\title{
Delayed diagnosis of Constructive Pericarditis Finalized Congested Hepatopathy: Anesthesia Management
}

\author{
Aykut A. ${ }^{1}$, Demir A. ${ }^{1}$, Kuşvuran Kurtay M. ${ }^{1}$, Sert D.E. ${ }^{2}$, Çatav Z. ${ }^{2}$ \\ ${ }^{I}$ Türkiye Yüksek Ihtisas Hospital, Dept Of Anaesthesiology, Ankara, Turkey, ${ }^{2}$ Türkiye Yüksek Ihtisas Hospital, \\ Dept Of Surgery, Ankara, Turkey
}

\section{Background}

Constrictive pericarditis is a clinical syndrome resulting from chronic inflammation, adhesions of the pericardial fibrotic calcification. Decreased cardiac compliance and diastolic dysfunction lead to systemic, hepatic and pulmonary venous congestion $(1,2)$. In this article, we aimed to present the perioperative anesthesia story of a case with a delayed diagnosis of cirrhosis due to constructive pericarditis.

\section{Case Report}

Forty-nine-year-old, $70 \mathrm{~kg}, 163 \mathrm{~cm}$, male patient, came to the hospital with doubt of constructive pericarditis.He was admitted to unconsciousness, shortness of breath and jaundice. He had intensive care with peripheral edema, ascites, $4 \mathrm{~cm}$ hepatomegaly and encephalopathy. In the tests antiHCV(+),Ammonia $420 \mathrm{mg} / \mathrm{dl}$.Bilirubin $2.5 \mathrm{mg} / \mathrm{dl}$,he was diagnosed as HCV cirrhosis + hepatic encephalopathy and gastrointestinal bleeding started during these examinations. When clinics tried to stabilize the patient, HCV was negative in the revised examinations. During this period, cardiology was consulted on the development of arrhythmia in the patient. In the echocardiographic, paradox in interventricular septal movements, ejection fraction 50\%, second degree mitraltricuspid insufficiency, Pulmonary artery-right atrium and right ventricular dilatation, systolic pulmonary artery pressure 60 $\mathrm{mmHg}$, vena cava inferior dilated, pericardium thick and bright, thoracic tomography was in favor of constructive pericarditis. The patient was operated on with high risk of neurological impairment with apathetic, slow-sense and responsive, partially oriented and moderate deceleration in EEG. Before Anesthesia induction, blood pressure was $114 / 60 \mathrm{mmHg}$, heart rate was 95 beats/min, right and left rSO2(serebral oxymetri with Nonin) values were 43/45, PSI(Patient state index with Sedline) 95, $\mathrm{SpO} 2$ 97. In anesthesia induction; ketamine, midazolam, lidocaine, fentanyl, rocuronium were used. Meanwhile, transamin and 5 mgkgmin-1 of dobutamine infusion was initiated. After induction, blood pressure was $118 / 64 \mathrm{mmHg}$, heart rate was 107 beats, right-left $\mathrm{rSO} 2$ values were 57/59, PSI 41, $\mathrm{SpO} 299 \%$ and $\mathrm{SEF}$ (spectral edge frequency) values were $6.6 / 6.5$ on the left. Central venous pressure was $26 \mathrm{mmHg}$. Transesophageal echocardiography was not wanted by surgery because the patient had esophageal varices. Mechanical ventilation which negatively affected the pulmonary system such as hyperoxia, hypercapnia and atelectasis was avoided. After sternotomy, the right cardiac structures were more predominantly calcified.The hemodynamics were stabilized using intravenous anesthesia according to PSI level.The pericardium was removed without cardiopulmonary bypass. There was no acute change in the function of the right heart regions after pericardiectomy, but the central venous pressure decreased to $19 \mathrm{mmHg}$. Because of the high pressure that is felt manually in pulmonary vascular structures intraoperatively, milrinone infusion was initiated. Dobutamine and milrinone accompanied the patient in intensive care. After 4 he was extubated and the infusions were terminated after 2 days.

\section{Discussion}

The left heart pulmonary venous return decreases during inspiration, but the venous return to the right heart continues. This, shifts the interventricular septum to the left and reduces the left ventricular cavity so that stroke volume is reduced. The most important compensatory response to decrease in stroke volume is the adrenergic stimulation(3). For this reason, it is important to maintain adrenergic stimulation in perioperative period in such patients. In addition, a decrease in baroreflex sensitivity has been shown as a component of cardiac cirrhosis, which is intertwined with many hemodynamic and biochemically important mechanisms and contributes to cardiac dysfunction(4). Therefore, to prevent adrenergic depression that may be caused by anesthesia induction, induction agents were combined with ketamine in this case and titrated at slowly increasing doses. Ketamine alone and high doses increase pulmonary vascular resistance, so it is more appropriate to use it in combination with midazolam and fentanyl. In order to adrenergic-inotropic and limit pulmonary vascular resistance to the patient, dobutamine infusion was started with anesthesia induction (3). Baseline NIRS values before anesthesia induction were $43 / 45$. Oxygen saturation and haemodynamic parameters are close to normal and it is difficult to say the exact reason for the low baseline $\mathrm{rSO} 2$ in this patient. However, after anesthesia induction, $\mathrm{rSO} 2$ values increased to 57/59 due to slowing of cerebral metabolism due to anesthetics. During operation, cerebral oxygenation values ranged from 55 to 60 and SEF ranged from 8 to $12 \mathrm{~Hz}$ and no pathology was observed. Since symptoms of encephalopathy persisted after the operation, the patient was followed up by neurology. In such patients with encephalopathy, neuromonitoring will help distinguish the neurological deficit from the new pathologies and provide anesthesia for cerebral protection

\section{References}

1. Braunwald's Heart Disease - A Textbook of Cardiovascular Medicine, Eugene Braunwald, W.B. Saunders Company; 6th Edition. 2. Manual of Cardiovascular Medicine- Eric J.Topol, Lippincott Williams \& Wilkins, 2004 3. Forrest P. Anaesthesia and right ventricular failure. Anaesth Intensive $\quad$ Care. 2009 May;37(3):370-85. 4. Ren X, Andrews AH. Cardiac cirrhosis. http://emedicine.medscape. com/article/151792-overview 